\title{
ANTISYMMETRY AND THE DIRECT INTEGRAL DECOMPOSITION OF UNSTARRED OPERATOR ALGEBRAS
}

\author{
WACLAW SZYMANSKI
}

\begin{abstract}
It is shown that the direct integral decomposition of a non-self-adjoint operator algebra $\mathscr{A}$ has the diagonal $\mathscr{A} \cap \mathscr{A}^{*}$ of this algebra as the algebra of diagonalizable operators if and only if almost all direct integrands of $\mathscr{A}$ are antisymmetric algebras. By using the antisymmetric decomposition a direct integral model of a commutative, reflexive algebra is obtained.
\end{abstract}

1. Introduction. The von Neumann reduction theory of $W^{*}$-algebras reduces the problem of investigating these algebras to the study of "the simplest" $W^{*}$-algebras, namely factors. In 1976 Azoff, Fong and Gilfeather [1] created a reduction theory for unstarred (i.e. non-self-adjoint) operator algebras. They introduced a direct integral decomposition of such algebras and proved several theorems that connect properties of a given unstarred operator algebra and of its direct integrands. In particular, they exhibited a dependence between the certain maximality property of the underlying diagonal algebra and the irreducibility of almost all direct integrands of a given algebra [1, Theorem 3.6]. This dependence, again, reduces the study of some general unstarred algebras to the study of "the simplest" algebras in this case, namely irreducible ones. But in the non-self-adjoint setting there is no unique idea-what does "simplest" really mean?

The present paper deals, in $\S 3$, with the case where "the simplest" algebras that occur in the AFG-direct integral decomposition of a given algebra are actually antisymmetric. $\$ 4$ gives a model of commutative, reflexive algebras of operators, and the set of all maximal antisymmetric projections for such algebras is described there in terms of their direct integral decomposition.

2. Preliminaries. Throughout this paper all Hilbert spaces are separable, all projections are self-adjoint, and all algebras of operators have unit. SOT denotes the strong operator topology in the algebra $L(H)$ of all linear, bounded operators in the Hilbert space $H$.

First the AFG-direct integral decomposition will be recalled. Fix an increasing sequence $H_{1} \subset H_{2} \subset \cdots \subset H_{\infty}$ of Hilbert spaces. Assume $\operatorname{dim} H_{n}=n$ for all finite $n$ and that $H_{\infty}$ is spanned by all the remaining $H_{n}$ 's. Let $\left(\Lambda, \mu,\left\{\sigma_{n}\right\}\right)$ be a partitioned measure space, i.e., $\Lambda$ is a separable metric space, $\mu$ is a regular, Borel, finite measure on $\Lambda$ and $\left\{\sigma_{n}\right\}$ is a Borel partition of $\Lambda$. The direct integral

Received by the editors March 8, 1985.

1980 Mathematics Subject Classification. Primary 46L15, 47B40, 46L45.

Key words and phrases. Direct integral decomposition, antisymmetric operator algebra, antisymmetric projection, atom of a measure. 
$\mathscr{H}=\int_{\Lambda}^{\oplus} H(\lambda) d \mu(\lambda)$ is defined as the Hilbert space consisting of all (equivalence classes of) functions $f: \Lambda \rightarrow H_{\infty}$ such that $f(\lambda) \in H(\lambda)=H_{n}$ for $\lambda \in \sigma_{n}$ and $\int_{\Lambda}\|f(\lambda)\|^{2} d \mu(\lambda)<\infty$. An operator $A \in L(\mathscr{H})$ is decomposable if there is a SOT-measurable function $A(\cdot): \Lambda \rightarrow L\left(H_{\infty}\right)$ such that $A(\lambda) \in L(H(\lambda)), \lambda \in \Lambda$, and $A f(\lambda)=A(\lambda) f(\lambda), \lambda \in \Lambda$ for all $f \in \mathscr{H}$. Such an operator is denoted by $A=\int_{\Lambda}^{\oplus} A(\lambda) d \mu(\lambda)$. An operator $A \in L(\mathscr{H})$ is diagonalizable if $A$ is decomposable and the values of the function $A(\cdot)$ are scalar multiples of the identity $I(\lambda)$ in $L(H(\lambda))$ for almost all $\lambda$. The commutative $W^{*}$-algebra $D$ of all diagonalizable operators is called the diagonal algebra of $\Lambda$. The principal property of this algebra is that it equals the commutant of the algebra of all decomposable operators. Moreover, the diagonal algebra of $\Lambda$ serves as a unitary model of every commutative $W^{*}$-algebra. Namely, to every commutative $W^{*}$-algebra $\mathscr{R} \subset L(H)$, there corresponds a unique partitioned measure space $\left(\Lambda, \mu,\left\{\sigma_{n}\right\}\right)$ such that $\mathscr{R}$ is unitarily equivalent to the diagonal algebra of $\Lambda$. The basic definitions and properties above belong to the classical von Neumann reduction theory and can be found in [3]. Now let $\left(\Lambda, \mu,\left\{\sigma_{n}\right\}\right)$ be a partitioned measure space with the diagonal algebra $D$. Let $\mathscr{A} \subset L(\mathscr{H})$ be a SOT-closed algebra of decomposable operators, i.e. $\mathscr{A} \subset D^{\prime}$ ( = the commutant of $D$ ). Choose a countable sequence $\left\{A_{n}\right\}$ of generators of $\mathscr{A}$. Since each $A_{n}$ is a decomposable operator, $A_{n}=\int_{\Lambda}^{\oplus} A_{n}(\lambda) d \mu(\lambda)$. Choose a Borel representative $\lambda \rightarrow A_{n}(\lambda)$ of the corresponding classes of functions. Let $\mathscr{A}(\lambda)$ be the SOT-closed algebra generated by $\left\{A_{n}(\lambda)\right\}$. Denote $\mathscr{A} \sim \int_{\Lambda}^{\oplus} \mathscr{A}(\lambda) d \mu(\lambda)$. This decomposition will be called the AFG-(direct integral) decomposition of the algebra with respect to $D$. The basic reference for the non-self-adjoint reduction theory is [1].

3. The role of antisymmetric algebras in the AFG-decomposition. Recall that an algebra $\mathscr{A} \subset L(H)$ is called antisymmetric if it contains no self-adjoint operators except scalar multiples of the identity. If $\mathscr{A}$ is SOT-closed, then $\mathscr{A}$ is antisymmetric if and only if the only projections in $\mathscr{A}$ are 0 or $I=$ the identity. The basic references for the antisymmetry in operator algebras are $[\mathbf{4}, \mathbf{6}]$. Let $\left(\Lambda, \mu,\left\{\sigma_{n}\right\}\right)$ be a partitioned measure space and let $D$ be the diagonal algebra of $\Lambda$. A simple observation that the projections $E_{n}=\int_{\Lambda}^{\oplus} \chi_{\sigma_{n}}(\lambda) I(\lambda) d \mu(\lambda)$ are diagonalizable and that they are mutually orthogonal allows one to considerably reduce the problem of study of the general direct integral. The following proposition is certainly well known. It is included here for the sake of completeness.

3.1. Proposition. Let $\mathscr{H}=\int_{\Lambda}^{\oplus} H(\lambda) d \mu(\lambda)$. Suppose that $\mathscr{A} \subset L(\mathscr{H})$ is an algebra such that $D \subset \mathscr{A} \subset D^{\prime}$. Then:

(i) $\mathscr{H}$ is unitarily equivalent to the Hilbert space $\oplus_{n=1}^{\infty} L_{H_{n}}^{2}\left(\sigma_{n}, \mu\right)$.

(ii) Each decomposable operator in $L(\mathscr{H})$ is unitarily equivalent to the direct sum of decomposable operators in $L_{H_{n}}^{2}\left(\sigma_{n}, \mu\right)$.

(iii) $\mathscr{A}=\bigoplus_{n=1}^{\infty} \mathscr{A}_{E_{n}}$.

Here $L_{H_{n}}^{2}\left(\sigma_{n}, \mu\right)=\left\{f: \sigma_{n} \rightarrow H_{n}: f\right.$ is measurable and $\left.\int_{\sigma_{n}}\|f(\lambda)\|^{2} d \mu(\lambda)<\infty\right\}$ and $\mathscr{A}_{E_{n}}$ consists of restrictions of operators in $\mathscr{A}$ to the space $E_{n} \mathscr{H}$. The proof of (i) and (ii) is clear. To see (iii) it is enough to notice that $E_{n} \in \mathscr{A} \cap \mathscr{A}^{\prime}$ for each $n$. 
The following theorem is the first main result of this paper. It explains the role of antisymmetric algebras in the AFG-decomposition.

3.2. Theorem. Suppose $D \subset L(H)$ is a commutative $W^{*}$-algebra. Consider $D$ to be the diagonal algebra of a partitioned measure space $\left(\Lambda, \mu,\left\{\sigma_{n}\right\}\right)$ in $\mathscr{H}=$ $\int_{\Lambda}^{\oplus} H(\lambda) d \mu(\lambda)$. Let $\mathscr{A} \subset L(\mathscr{H})$ be a SOT-closed algebra. Suppose $D \subset \mathscr{A} \subset D^{\prime}$. Let $\mathscr{A} \sim \int_{\Lambda}^{\oplus} \mathscr{A}(\lambda) d \mu(\lambda)$ be the AFG-decomposition of $\mathscr{A}$ with respect to $D$. Then $D=\mathscr{A} \cap \mathscr{A}^{*}$ if and only if almost all $\mathscr{A}(\lambda)$ are antisymmetric.

Proof. Assume first that almost all $\mathscr{A}(\lambda)$ are antisymmetric. Clearly, $\mathscr{A}^{*}=\left\{A^{*}\right.$ : $A \in \mathscr{A}\}$. Since $D \subset \mathscr{A}$ by the assumption, and since $D$ is a $W^{*}$-algebra, it follows that $D \subset \mathscr{A} \cap \mathscr{A}^{*}$. The algebra $\mathscr{A} \cap \mathscr{A}^{*}$ is a $W^{*}$-algebra generated by self-adjoint elements of $\mathscr{A}$. Take $S=S^{*} \in \mathscr{A}$. Since $\mathscr{A}$ consists of decomposable operators $\left(\mathscr{A} \subset D^{\prime}\right), \quad S$ is decomposable. Thus $S=\int_{\Lambda}^{\oplus} S(\lambda) d \mu(\lambda)$. Almost all $S(\lambda)$ are self-adjoint operators in $H(\lambda)$. Moreover, $S(\lambda) \in \mathscr{A}(\lambda)$ for almost all $\lambda$ by Proposition 3.3 of [1]. Since almost all $\mathscr{A}(\lambda)$ are assumed to be antisymmetric, $S(\lambda)$ are scalar multiples of the identities, i.e., there are $c(\lambda) \in R$ such that $S(\lambda)=c(\lambda) I(\lambda)$ for almost all $\lambda$. The function $\lambda \rightarrow c(\lambda)$ is an $L^{\infty}(\mu)$-function. Therefore $S$ is a diagonalizable operator. Thus all the generators of the $W^{*}$-algebra $\mathscr{A} \cap \mathscr{A}^{*}$ belong to $D$. Hence $\mathscr{A} \cap \mathscr{A}^{*} \subset D$.

Now assume $D=\mathscr{A} \cap \mathscr{A}^{*}$. Suppose additionally that all the $H(\lambda)$ are equal, i.e. $H(\lambda)=K$ for all $\lambda$. Once the assertion of the theorem is proved for this case, Proposition 3.1 extends it immediately to the general case. Define $\eta=\{(\lambda, Q) \in \Lambda$ $\times$ ball $L(K): Q$ is a projection, $Q \neq 0, Q \neq I, Q \in \mathscr{A}(\lambda)\} . \eta$ is a Borel relation in $\Lambda \times$ ball $L(K)$, where ball $L(K)$ is the unit ball in $L(K)$ equipped with SOT. The domain $\sigma$ of $\eta$ is the set of all $\lambda \in \Lambda$ such that $\mathscr{A}(\lambda)$ contains a nontrivial projection. This amounts to saying that $\sigma=\{\lambda \in \Lambda: \mathscr{A}(\lambda)$ is not antisymmetric $\}$, by the remark after the definition of an antisymmetric algebra. By the Principle of Measurable Choice (see e.g. [1, Proposition 2.1]), $\sigma$ is measurable and there is a Borel function $\tilde{P}(\cdot)$ whose domain almost coincides with $\sigma$ and whose graph is contained in $\eta$. Define the function $P(\lambda)=\tilde{P}(\lambda)$ for $\lambda$ in the domain of $\tilde{P}(\cdot)$ and $P(\lambda)=0$ for $\lambda \in \Lambda$ not in the domain of $P(\cdot)$. Then $P(\cdot)$ is a SOT-measurable function and $\sup \{\|P(\lambda)\|: \lambda \in \Lambda\} \leqslant 1$. Let $P=\int_{\Lambda}^{\oplus} P(\lambda) d \mu(\lambda)$. Then $P$ is a decomposable projection and $P$ belongs to $\mathscr{A}$ by Proposition 3.3 of [1], because $P(\lambda) \in \mathscr{A}(\lambda)$ for almost all $\lambda$. Since $\mathscr{A} \cap \mathscr{A}^{*}=D$, it follows that all the projections in $\mathscr{A}$ are diagonalizable. Therefore $P(\lambda)=0$ or $P(\lambda)=I$ for almost all $\lambda$. This proves that $\mu(\sigma)=0$. Thus almost all $\mathscr{A}(\lambda)$ are antisymmetric. Q.E.D.

4. Direct integral and maximal antisymmetric projections for reflexive algebras. In the previous section the "global" antisymmetry was considered. Now the AFGdecomposition will be employed to get a direct integral decomposition for commutative, reflexive algebras, which allows us to determine completely the set of all maximal antisymmetric projections for such algebras.

The concept of an antisymmetric projection was introduced in [4] as a noncommutative generalization of the notion of a set of antisymmetry for function algebras. Let $\mathscr{A} \subset L(H)$ be an algebra of operators. A projection $E \in \mathscr{A}^{\prime}$ is called an 
antisymmetric projection for $\mathscr{A}$ if the algebra $\mathscr{A}_{E}=\{A \mid \mathrm{EH}: A \in \mathscr{A}\} \subset L(\mathrm{EH})$ is antisymmetric. An antisymmetric projection for $\mathscr{A}$ is called a maximal antisymmetric projection for $\mathscr{A}$ if it is a maximal (in the usual ordering of projections) element in the family of all antisymmetric projections for $\mathscr{A}$. The set of all maximal antisymmetric projections for $\mathscr{A}$ is denoted by $\mathscr{M}(\mathscr{A})$. The elements of $\mathscr{M}(\mathscr{A})$ are mutually orthogonal. The projection $I-\sum\{F: F \in \mathscr{M}(\mathscr{A})\}$ is called the pseudosymmetric projection for $\mathscr{A}$. The reader is referred to $[4,6]$ for details concerning antisymmetric projections. In particular, the following theorem was proved in [4].

4.1. Theorem ([4, Corollary 2]). If $\mathscr{A} \subset L(H)$ is a commutative, reflexive algebra, then $\mathscr{A}$ admits the antisymmetric decomposition, i.e., $\mathscr{M}(\mathscr{A}) \subset \mathscr{A} \cap \mathscr{A}^{\prime}$, i.e. 4.2. $\mathscr{A}=\mathscr{A}_{0} \oplus\left(\oplus\left\{\mathscr{A}_{F}: F \in \mathscr{M}(\mathscr{A})\right\}\right)$, where $\mathscr{A}_{0}$ is the pseudosymmetric part of $\mathscr{A}$.

Let $\mathscr{A} \subset L(H)$ be an algebra. A projection $P \in \mathscr{A}$ is called minimal in $\mathscr{A}$ if for each projection $Q \in \mathscr{A}, Q \leqslant P$ either $Q=0$ or $Q=P$. It can be easily deduced from Proposition 2.1 of [5] that

4.3. If $\mathscr{A} \subset L(H)$ is a commutative, reflexive algebra, then $\mathscr{M}(\mathscr{A})$ equals the set of all minimal projections in $\mathscr{A}$. Let $\mathscr{A} \subset L(H)$ be a commutative, reflexive algebra. All the projections in $\mathscr{A}$ are, clearly, in the commutative $W^{*}$-algebra $\mathscr{A} \cap \mathscr{A}^{*}$. Therefore, minimal projections in $\mathscr{A}$ are exactly minimal projections in $\mathscr{A} \cap \mathscr{A}^{*}$. Thus, by 4.3 , the antisymmetric decomposition 4.2 depends only upon the algebra $\mathscr{A} \cap \mathscr{A}^{*}$, not on the algebra $\mathscr{A}$ itself. This proves the following:

4.4. Proposition. Suppose $\mathscr{A}, \mathscr{B} \subset L(H)$ are two reflexive, commutative algebras. If $\mathscr{A} \cap \mathscr{A}^{*}=\mathscr{B} \cap \mathscr{B}^{*}$, then $\mathscr{M}(\mathscr{A})=\mathscr{M}(\mathscr{B})=\mathscr{M}\left(\mathscr{A} \cap \mathscr{A}^{*}\right)=$ the set of all minimal projections in $\mathscr{A} \cap \mathscr{A}^{*}$.

Notice that the last two equalities are a consequence of the reflexivity of the von Neumann algebra $\mathscr{A} \cap \mathscr{A}^{*}$.

Now everything is prepared to give the description of maximal antisymmetric projections for commutative, reflexive algebras.

4.5. TheOREM. Let $\mathscr{A} \subset L(H)$ be a commutative, reflexive algebra. Put $D=\mathscr{A} \cap$ $\mathscr{A}^{*}$. Let $\left(\Lambda, \mu,\left\{\sigma_{n}\right\}\right)$ be the partitioned measure space such that $D$ is unitarily equivalent to the diagonal algebra of $\Lambda . H$ is unitarily equivalent to $\int_{\Lambda}^{\oplus} H(\lambda) d \mu(\lambda)$. Then:

(i) $\mathscr{A}$ is unitarily equivalent to a commutative, reflexive algebra of decomposable operators.

(ii) There is a measurable partition $\left\{\omega_{n}: n=0,1,2, \ldots\right\}$ of $\Lambda$ such that each $\omega_{n}$ is an atom of the measure $\mu$ for $n=1,2, \ldots$ and $\omega_{0}=\Lambda-\bigcup_{n=1}^{\infty} \omega_{n}$.

(iii) The correspondence $\omega_{n} \rightarrow F_{n}=\int_{\Lambda}^{\oplus} \chi_{\omega_{n}}(\lambda) I(\lambda) d \mu(\lambda)$ for $n=1,2, \ldots$ is oneto-one and it maps the set of atoms of the measure $\mu$ onto the set of all maximal antisymmetric projections for $\mathscr{A}$.

(iv) The projection $F_{0}=\int_{\Lambda}^{\oplus} \chi_{\omega_{0}}(\lambda) I(\lambda) d \mu(\lambda)$ is the pseudosymmetric projection for $\mathscr{A}$.

(v) $\mathscr{A}=\mathscr{A}_{F_{0}} \oplus\left(\oplus_{n=1}^{\infty} \mathscr{A}_{F_{n}}\right)$. 
Proof. Let the algebra $D$ be treated as the algebra of all diagonalizable operators and let $\mathscr{A}$ be considered as unitarily embedded into $L\left(\int_{\Lambda}^{\oplus} H(\lambda) d \mu(\lambda)\right)$. Since $\mathscr{A}$ is commutative, $\mathscr{A} \subset \mathscr{A}^{\prime}$. Therefore $D \subset \mathscr{A} \subset \mathscr{A}^{\prime} \subset D^{\prime}$. Thus $\mathscr{A}$ consists of decomposable operators. Clearly, the reflexivity is preserved by unitary isomorphisms. This proves (i). Now look for the set of minimal projections in $D$, which, by 4.4 , coincides with $\mathscr{M}(\mathscr{A})$. If $P \in D$ is a projection, then it is diagonalizable. Thus there is a measurable set $\omega \subset \Lambda$ such that $P=\int_{\Lambda}^{\oplus} \chi_{\omega}(\lambda) I(\lambda) d \mu(\lambda)$. It follows easily from the properties of direct integrals that $P$ is minimal in $D$ if and only if $\omega$ is an atom of $\mu$. Since $\mathscr{M}(\mathscr{A}) \subset D$, by 4.3 , to each $F \in \mathscr{M}(\mathscr{A})$ there corresponds a uniquely determined (up to measure zero) measurable set $\omega \subset \Lambda$, which is an atom of the measure $\mu$, such that $F=\int_{\Lambda}^{\oplus} \chi_{\omega}(\lambda) I(\lambda) d \mu(\lambda)$. Since elements of $\mathscr{M}(\mathscr{A})$ are mutually orthogonal projections [4, Proposition 3], the corresponding atoms have to be pairwise disjoint (up to measure zero). But there can be at most countably many of these atoms, because $\mu$ is a finite measure. This, together with 4.1 , proves the remaining assertions of the theorem. Q.E.D.

This theorem can be treated as a noncommutative Bishop decomposition theorem for commutative, reflexive algebras. It has to be pointed out that Theorem 4.5 as well as all the considerations in this section have a noncommutative character, because nothing is assumed about the commutativity of the $W^{*}$-algebra generated by $\mathscr{A}$. The reader may find more comments on this in $[4,6]$.

A remarkable similarity of Theorem 4.5 to Theorem 3.1 of [2] is apparent here. But it is easily seen that the situation in [2] is much more particular than the one treated in Theorem 4.5. The great difference between these two situations was already explained in detail in [4]. Nevertheless, a theorem like 4.5 cannot be proved without the direct integral theory. Thus, the similarity mentioned above is, clearly, mostly a matter of form.

\section{REFERENCES}

1. E. A. Azoff, C. K. Fong and F. Gilfeather, A reduction theory for non-self-adjoint operator algebras, Trans. Amer. Math. Soc. 224 (1976), 351-366.

2. J. B. Conway and R. F. Olin, A functional calculus for subnormal operators, Mem. Amer. Math. Soc., Vol. 10, No. 184 (1977).

3. J. T. Schwartz, $W^{*}$-algebras, Gordon and Breach, New York, 1967.

4. W. Szymanski, Antisymmetric operator algebras. II, Ann. Polon. Math. 37 (1980), 299-311.

5. Antisymmetry in the WOT-closed algebra generated by an isometri, Bull. Acad. Polon. Sci. Sér. Sci. Math. 28 (1980), 137-142.

6. __ Antisymmetry in operator algebras, Operator Algebras and Group Representations, Vol. II, Pitman, New York, 1982, pp. 190-197.

Department of Applied Mathematics, Hugo Kollataj Academy, Krakow, Poland

DePartment of Mathematics, Indiana University, BloOMINgton, Indiana 47405

Current address: Department of Mathematical Sciences, West Chester University, West Chester, Pennsylvania 19383 